\title{
SHIFTED QUADRATIC ZETA SERIES
}

\section{ANTHONY SOFO}

Received 3 February 2004 and in revised form 30 June 2004

It is well known that the Riemann Zeta function $\zeta(p)=\sum_{n=1}^{\infty} 1 / n^{p}$ can be represented in closed form for $p$ an even integer. We will define a shifted quadratic Zeta series as $\sum_{n=1}^{\infty} 1 /\left(4 n^{2}-\alpha^{2}\right)^{p}$. In this paper, we will determine closed-form representations of shifted quadratic Zeta series from a recursion point of view using the Riemann Zeta function. We will also determine closed-form representations of alternating sign shifted quadratic Zeta series.

2000 Mathematics Subject Classification: 40C99, 40D99.

1. Introduction. In this paper, we will define a shifted quadratic Zeta series as one of the form

$$
S(a, p):=\sum_{n=1}^{\infty} \frac{1}{\left(4 n^{2}-(2 a+1)^{2}\right)^{p}},
$$

where $p$ is a positive integer and $a=0,1,2, \ldots$.

The Riemann Zeta function, $\zeta(p)$ is defined by

$$
\zeta(p)=\sum_{n=1}^{\infty} \frac{1}{n^{p}}, \quad \mathbb{R}(p)>1,
$$

and we will also define

$$
\delta(p):=\sum_{n=1}^{\infty} \frac{1}{(2 n-1)^{p}} .
$$

The alternating sign series version of (1.1) will be defined as

$$
A S(a, p):=\sum_{n=1}^{\infty} \frac{(-1)^{n+1}}{\left(4 n^{2}-(2 a+1)^{2}\right)^{p}}
$$

for $p$, a positive integer, and $a=0,1,2, \ldots$.

The Dirichlet series, $D(p)$ is defined as

$$
D(p):=\sum_{n=1}^{\infty} \frac{(-1)^{n+1}}{n^{p}}, \quad \mathbb{R}(p)>1,
$$


and furthermore, we define

$$
\sigma(p):=\sum_{n=1}^{\infty} \frac{(-1)^{n+1}}{(2 n-1)^{p}} .
$$

The following formulae for $\zeta(p)$ have also been given.

Euler, in 1748 gave the formula

$$
\zeta(2 q)=\sum_{n=1}^{\infty} \frac{1}{n^{2 q}}=\frac{(-1)^{q-1} 2^{2 q-1} \pi^{2 q}}{(2 q) !} B_{2 q},
$$

where $B_{2 q}$ denotes Bernoulli numbers for $q \in \mathbb{N}$. The Bernoulli and Euler numbers, $B_{q}$ and $E_{q}$ are defined, respectively, by

$$
\begin{array}{ll}
\frac{t}{e^{t}-1}=\sum_{j=0}^{\infty} \frac{t^{j}}{j !} B_{j}, & |t|<2 \pi, \\
\frac{2 e^{t}}{e^{t}+1}=\sum_{j=0}^{\infty} \frac{t^{j}}{j !} E_{j}, & |t| \leq \pi .
\end{array}
$$

Lin [9] in 1999 gave the following elementary expression for $\zeta(2 q)$ : let $q \in \mathbb{N}$, then

$$
\sum_{n=1}^{\infty} \frac{1}{n^{2 q}}=K_{q} \pi^{2 q}
$$

where $K_{q}$ is given by the recurrence relation

$$
K_{q}=\frac{(-1)^{q-1} q}{(2 q+1) !}-\sum_{j=1}^{q-1} \frac{(-1)^{q-j}}{(2 q-2 j+1) !} K_{j} .
$$

The main aim of this paper is to determine closed form representations of $S(a, p)$ in terms of $\delta(p)$ and the Riemann Zeta function $\zeta(p)$. The closed form of $A S(a, p)$ will also be given. It is well known that $\zeta(p)$ can be represented in closed form for $p$ an even integer, although no closed representation of $\zeta(p)$ exists for $p$ an odd integer. Closed form representations of $S(a, p), \delta(p)$, and $A S(a, p)$ for particular cases of $a$ and $p$ can be determined from contour integral methods and the interested reader is referred to the excellent paper by Flajolet and Salvy [4].

Luo et al. [10] obtained the following three theorems, expressing (1.2), (1.5), and (1.6) as a recurrence relation, from the point of view of Fourier series analysis.

THEOREM 1.1. For $q \in \mathbb{N}$,

$$
\begin{gathered}
\zeta(2 q)=\frac{(-1)^{q-1} q \pi^{2 q}}{(2 q+1) !}-\sum_{j=1}^{q-1} \frac{(-1)^{q-j} \pi^{2 q-2 j}}{(2 q-2 j+1) !} \zeta(2 j), \\
\zeta(2 q+1)=\frac{2^{2 q+1}}{2^{2 q+1}-1}\left[2 \sum_{n=1}^{\infty} \frac{1}{(4 n-1)^{2 q+1}}+\sigma(2 q+1)\right] .
\end{gathered}
$$


THEOREM 1.2. For $q \in \mathbb{N}$, the following hold:

$$
\begin{aligned}
\zeta(2 q) & =\frac{(-1)^{q-1} 2^{2 q-1} \pi^{2 q}}{(2 q) !} B_{2 q}, \\
\zeta(2 q+1) & =\frac{\pi^{2 q+1} E_{q}}{\left(2^{2 q+2}-2\right)(2 q) !}+\frac{2^{2 q+2}}{2^{2 q+1}-1} \sum_{n=1}^{\infty} \frac{1}{(4 n-1)^{2 q+1}},
\end{aligned}
$$

where $B_{j}$ and $E_{j}$ are the Bernoulli and Euler numbers defined by (1.8) and (1.9).

For the alternating case, the following holds.

THEOREM 1.3. For $q \in \mathbb{N}$,

$$
\begin{gathered}
D(2 q)=\frac{(-1)^{q-1} \pi^{2 q}}{2(2 q+1) !}-\sum_{j=1}^{q-1} \frac{(-1)^{q+j} \pi^{2 q-2 j}}{(2 q-2 j+1) !} D(2 j), \\
\sigma(2 q+1)=\frac{(-1)^{q} \pi^{2 q+1}}{2^{2 q+2}(2 q+1) !}-\sum_{j=1}^{q-1} \frac{(-1)^{q+j} \pi^{2 q-2 j}}{(2 q-2 j+1) !} \sigma(2 j+1) .
\end{gathered}
$$

Additionally, in particular, cases (1.1), (1.3), and (1.4) can be determined in closed form by Fourier series analysis.

The Fourier series representation

$$
\sum_{n=1}^{\infty} \frac{\cos 2 n x}{\left(4 n^{2}-1\right)}=\frac{1}{2}-\frac{\pi}{4} \sin x, \quad x \in\left[0, \frac{\pi}{2}\right],
$$

leads to the result (1.1) and (1.4) for $a=0$ and $p=1$.

In a similar way, the Fourier series representation

$$
\sum_{n=1}^{\infty} \frac{\cos (2 n-1) x}{(2 n-1)^{2}}=\frac{\pi}{4}\left(\frac{\pi}{2}-|x|\right), \quad x \in[-\pi, \pi],
$$

leads to the closed form representation of (1.3) for $p=2$.

Hence the development of a recurrence formula for $S(a, p)$ in terms of the Riemann Zeta function, $\zeta(p)$, has the advantage, over contour integral methods and Fourier series analysis, of simplicity in determining closed form representations of $S(a, p)$ for any integer values $a$ and $p$.

The next two lemmas will be useful in the proof of the main results in this paper.

2. Quadratic nonalternating case. The following lemma will be required later.

LEMMA 2.1. For $a=0,1,2, \ldots$ and $p$ a positive integer $\geq 2$,

(i)

$$
\delta(p)=\left(1-\frac{1}{2^{p}}\right) \zeta(p)
$$


(ii)

$$
\sum_{n=1}^{\infty} \frac{1}{(2 n-2 a-1)^{p}}=\delta(p)+\sum_{r=1}^{a} \frac{1}{(2 r-2 a-1)^{p}},
$$

(iii)

$$
\begin{aligned}
& \sum_{n=1}^{\infty} \frac{(2 n+2 a+1)^{p}-(2 n-2 a-1)^{p}}{\left(4 n^{2}-(2 a+1)^{2}\right)^{p}} \text { for } p \text { odd, } \\
&=\sum_{r=1}^{2 a+1} \frac{1}{(2 r-2 a-1)^{p}}=\frac{1}{(2 a+1)^{p}}+ \begin{cases}0, & \text { for } p \text { even, } \\
\sum_{r=1}^{a} \frac{2}{(2 a+1-2 r)^{p}} & \text {, }\end{cases}
\end{aligned}
$$

(iv)

$$
\sum_{n=1}^{\infty} \frac{1}{(2 n+2 a+1)^{p}}=\delta(p)-\sum_{r=0}^{a} \frac{1}{(2 r+1)^{p}},
$$

(v) for $p=1$,

$$
\begin{aligned}
\sum_{n=1}^{\infty} \frac{2(2 a+1)}{(2 n-2 a-1)(2 n+2 a+1)} & =\sum_{n=1}^{\infty}\left(\frac{1}{2 n-2 a-1}-\frac{1}{2 n+2 a+1}\right) \\
& =\sum_{n=1}^{\infty}\left(\frac{1}{2 n-1}-\frac{1}{2 n-1}\right)+\frac{1}{2 a+1}=\frac{1}{2 a+1} .
\end{aligned}
$$

Proof. (i) follows directly upon subtracting

$$
\sum_{n=1}^{\infty} \frac{1}{(2 n)^{p}}
$$

from $\zeta(p)$.

(ii)

$$
\begin{aligned}
\sum_{n=1}^{\infty} \frac{1}{(2 n-2 a-1)^{p}} & =\frac{1}{(1-2 a)^{p}}+\frac{1}{(3-2 a)^{p}}+\cdots+\frac{1}{(-3)^{p}}+\frac{1}{(-1)^{p}}+\frac{1}{1^{p}}+\frac{1}{3^{p}}+\cdots \\
& =\sum_{r=1}^{a} \frac{1}{(2 r-2 a-1)^{p}}+\delta(p) .
\end{aligned}
$$

(iii)

$$
\begin{aligned}
\sum_{n=1}^{\infty} \frac{1}{(2 n-(2 a+1))^{p}}= & \frac{1}{(1-2 a)^{p}}+\frac{1}{(3-2 a)^{p}}+\cdots+\frac{1}{(-3)^{p}}+\frac{1}{(-1)^{p}}+1+\frac{1}{3^{p}}+\cdots \\
& +\frac{1}{(2 a-1)^{p}}+\frac{1}{(2 a+1)^{p}}+\frac{1}{(2 a+3)^{p}}+\cdots, \\
\sum_{n=1}^{\infty} \frac{1}{(2 n+(2 a+1))^{p}}= & \frac{1}{(3+2 a)^{p}}+\frac{1}{(5+2 a)^{p}}+\frac{1}{(7+2 a)^{p}}+\cdots,
\end{aligned}
$$


by subtraction

$$
\begin{aligned}
& \sum_{n=1}^{\infty}\left\{\frac{1}{(2 n-(2 a+1))^{p}}-\frac{1}{(2 n+(2 a+1))^{p}}\right\} \\
& =\frac{1}{(1-2 a)^{p}}+\frac{1}{(3-2 a)^{p}}+\cdots+\frac{1}{(2 a-3)^{p}}+\frac{1}{(2 a-1)^{p}}+\frac{1}{(2 a+1)^{p}}, \\
& \sum_{n=1}^{\infty} \frac{(2 n+(2 a+1))^{p}-(2 n-(2 a+1))^{p}}{\left(4 n^{2}-(2 a+1)^{2}\right)^{p}} \\
& =\sum_{r=1}^{2 a+1} \frac{1}{(2 r-(2 a+1))^{p}}=\frac{1}{(2 a+1)^{p}}+\sum_{r=1}^{a} \frac{1+(-1)^{p}}{(2 a-(2 r-1))^{p}} \\
& =\frac{1}{(2 a+1)^{p}}+ \begin{cases}0, & \text { for } p \text { odd }, \\
\sum_{r=1}^{a} \frac{2}{(2 a-(2 r-1))^{p}} & \text { for } p \text { even. }\end{cases}
\end{aligned}
$$

(iv) From part (iii) we may write

$$
\sum_{n=1}^{\infty} \frac{1}{(2 n+(2 a+1))^{p}}=\sum_{n=1}^{\infty} \frac{1}{(2 n-(2 a+1))^{p}}-\sum_{r=1}^{2 a+1} \frac{1}{(2 r-(2 a+1))^{p}},
$$

and from part (ii),

$$
\begin{aligned}
\sum_{n=1}^{\infty} \frac{1}{(2 n+(2 a+1))^{p}} & =\sum_{n=1}^{\infty} \frac{1}{(2 n-1)^{p}}+\sum_{r=1}^{a} \frac{1}{(2 r-(2 a+1))^{p}}-\sum_{r=1}^{2 a+1} \frac{1}{(2 r-(2 a+1))^{p}} \\
& =\sum_{n=1}^{\infty} \frac{1}{(2 n-1)^{p}}-\sum_{r=a+1}^{2 a+1} \frac{1}{(2 r-(2 a+1))^{p}} \\
& =\sum_{n=1}^{\infty} \frac{1}{(2 n-1)^{p}}-\sum_{r=1}^{a+1} \frac{1}{(2 r-1)^{p}} \\
& =\delta(p)-\sum_{r=0}^{a} \frac{1}{(2 r+1)^{p}} .
\end{aligned}
$$

(v) For the case $p=1$, we have

$$
\sum_{n=1}^{\infty} \frac{2(2 a+1)}{(2 n-2 a-1)(2 n+2 a+1)}=\sum_{n=1}^{\infty}\left(\frac{1}{2 n-2 a-1}-\frac{1}{2 n+2 a+1}\right) .
$$

Let

$$
u_{n}=\frac{1}{2 n-2 a-1}-\frac{1}{2 n+2 a+1},
$$

and note that there are only a finite number of negative terms, for $n \leq a$, in the first part of the expression for $u_{n}$. Now

$$
u_{n}=\frac{1}{2 n-2 a-1}-\frac{1}{2 n+2 a+1}=\frac{2(2 a+1)}{(2 n-2 a-1)(2 n+2 a+1)} \sim \frac{2 a+1}{2 n^{2}}=v_{n} .
$$


Since $\sum_{n=1}^{\infty} v_{n}$ is a convergent $p(p=2)$ series, it follows by the comparison test that $\sum_{n=1}^{\infty} u_{n}$ converges, see [3]. Moreover, by telescoping of the series,

$$
\begin{aligned}
\sum_{n=1}^{\infty} \frac{2(2 a+1)}{(2 n-2 a-1)(2 n+2 a+1)}= & \sum_{n=1}^{\infty}\left(\frac{1}{2 n-2 a-1}-\frac{1}{2 n+2 a+1}\right) \\
= & \sum_{n=1}^{\infty}\left(\frac{1}{2 n-1}+\sum_{r=1}^{a} \frac{1}{2 r-2 a-1}-\frac{1}{2 n-1}+\sum_{r=1}^{a+1} \frac{1}{2 r-1}\right) \\
= & \sum_{n=1}^{\infty}\left(\frac{1}{2 n-1}-\frac{1}{2 n-1}\right)+\frac{1}{2 a+1} \\
& +\sum_{r=1}^{a}\left(\frac{1}{2 r-2 a-1}+\frac{1}{2 r-1}\right) \\
= & \frac{1}{2 a+1} .
\end{aligned}
$$

Hence the lemma is proved.

LEMMA 2.2. For $p=1,2,3, \ldots, a \geq 0$ and

$$
A_{j}=\lim _{x \rightarrow(2 a+1) / 2} \frac{1}{(j-1) ! 2^{p+j-1}} \frac{d^{j-1}}{d x^{j-1}}\left[\left(x-\frac{2 a+1}{2}\right)^{p} F(x)\right] j=1,2, \ldots, p,
$$

where

$$
F(x)=\frac{1}{\left(x^{2}-((2 a+1) / 2)^{2}\right)^{p}},
$$

then

$$
\sum_{j=1}^{p} \frac{\left|A_{j}\right|}{(2 a+1)^{p-j+1}}=\frac{1}{2(2 a+1)^{2 p}} .
$$

Proof. For

$$
\begin{aligned}
& j=1, \quad\left|A_{1}\right|=\frac{1}{0 ! 2^{p}(2 a+1)^{p}}, \\
& j=2, \quad\left|A_{2}\right|=\frac{p}{1 ! 2^{p+1}(2 a+1)^{p+1}}, \\
& \vdots \\
& j=p, \quad\left|A_{p}\right|=\frac{p(p+1) \cdots(p+p-2)}{(p-1) ! 2^{p+p-1}(2 a+1)^{p+p-1}},
\end{aligned}
$$


then

$$
\begin{aligned}
\sum_{j=1}^{p} \frac{\left|A_{j}\right|}{(2 a+1)^{p-j+1}} & =\frac{1}{0 ! 2^{p}(2 a+1)^{p}}+\frac{p}{1 ! 2^{p+1}(2 a+1)^{p+1}}+\cdots+\frac{p(p+1) \cdots(p+p-2)}{(p-1) ! 2^{2 p-1}(2 a+1)^{2 p}} \\
& =\frac{1}{(2 a+1)^{2 p}} \sum_{j=1}^{p} \frac{(p+j-2) !}{(j-1) ! 2^{p+j-1}(p-1) !} \\
& =\frac{1}{(2 a+1)^{2 p}} \sum_{j=1}^{p} \frac{1}{2^{p+j-1}}\left(\begin{array}{c}
p+j-2 \\
j-1
\end{array}\right) \\
& =\frac{1}{(2 a+1)^{2 p}} \cdot \frac{1}{2^{p}} \cdot 2^{p-1} \\
& =\frac{1}{2(2 a+1)^{2 p}}
\end{aligned}
$$

hence the lemma is proved.

We now state and prove the main theorem for the quadratic nonalternating case.

THEOREM 2.3. For $p$, a positive integer, and $a \in \mathbb{N} \cup\{0\}$,

$$
S(a, p)=\frac{(-1)^{p+1}}{2(2 a+1)^{2 p}}+(-1)^{p} \sum_{k=1}^{p}\left|A_{p-k+1}\right|\left(1+(-1)^{k}\right)\left(1-\frac{1}{2^{k}}\right) \zeta(k),
$$

where $A_{j}$ is defined by (2.16) and $\zeta(k)$ by (1.2).

Proof. We may write

$$
\sum_{n=1}^{\infty} \frac{1}{\left(4 n^{2}-(2 a+1)^{2}\right)^{p}}=\sum_{n=1}^{\infty} \sum_{j=1}^{p}\left[\frac{A_{j}}{(2 n-(2 a+1))^{p-j+1}}+\frac{B_{j}}{(2 n+(2 a+1))^{p-j+1}}\right],
$$

where $A_{j}$ is given by (2.16) and similarly

$$
B_{j}=\lim _{x \rightarrow-(2 a+1) / 2} \frac{1}{(j-1) ! 2^{p+j-1}} \frac{d^{j-1}}{d x^{j-1}}\left[\left(x+\frac{2 a+1}{2}\right)^{p} F(x)\right], \quad j=1,2, \ldots, p .
$$

Now $A_{j}$ and $B_{j}$ are related by

$$
A_{j}=(-1)^{j+1}\left|A_{j}\right|, \quad B_{j}=(-1)^{p}\left|A_{j}\right| .
$$

So if we wish, $B_{j}=(-1)^{p+j+1}\left|A_{j}\right|$. 
Using (2.24), we can now write

$$
\begin{aligned}
\sum_{n=1}^{\infty} \frac{1}{\left(4 n^{2}-(2 a+1)^{2}\right)^{p}} & =\sum_{n=1}^{\infty} \sum_{j=1}^{p}\left[\frac{(-1)^{j+1}\left|A_{j}\right|}{(2 n-(2 a+1))^{p-j+1}}+\frac{(-1)^{p}\left|A_{j}\right|}{(2 n+(2 a+1))^{p-j+1}}\right] \\
& =\sum_{j=1}^{p}\left|A_{j}\right| \sum_{n=1}^{\infty}\left[\frac{(-1)^{j+1}}{(2 n-(2 a+1))^{p-j+1}}+\frac{(-1)^{p}}{(2 n+(2 a+1))^{p-j+1}}\right],
\end{aligned}
$$

upon interchanging the summation.

By telescoping of the series, from Lemma 2.1, (2.2), (2.4), and (2.1), we have

$$
\begin{aligned}
\sum_{n=1}^{\infty} & \frac{1}{\left(4 n^{2}-(2 a+1)^{2}\right)^{p}} \\
= & \sum_{j=1}^{p}\left|A_{j}\right| \sum_{n=1}^{\infty}\left[\frac{(-1)^{j+1}}{(2 n-1)^{p-j+1}}+\frac{(-1)^{p}}{(2 n-1)^{p-j+1}}\right. \\
& \left.+\sum_{r=1}^{a} \frac{(-1)^{j+1}}{(2 r-(2 a+1))^{p-j+1}}-\sum_{r=0}^{a} \frac{(-1)^{p}}{(2 r+1)^{p-j+1}}\right] \\
= & \sum_{j=1}^{p}\left|A_{j}\right|\left[\sum_{r=1}^{a} \frac{(-1)^{j+1}}{(2 r-(2 a+1))^{p-j+1}}+\sum_{r=0}^{a} \frac{(-1)^{p+1}}{(2 r+1)^{p-j+1}}\right] \\
& +\sum_{j=1}^{p}\left|A_{j}\right| \sum_{n=1}^{\infty}\left[\frac{(-1)^{j+1}}{(2 n-1)^{p-j+1}}+\frac{(-1)^{p}}{(2 n-1)^{p-j+1}}\right] \\
= & \sum_{j=1}^{p} \frac{(-1)^{p+1}\left|A_{j}\right|}{(2 a+1)^{p-j+1}}+\sum_{j=1}^{p}\left|A_{j}\right| \sum_{r=1}^{a}\left[\frac{(-1)^{j+1}}{(2 r-(2 a+1))^{p-j+1}}+\frac{(-1)^{p+1}}{(2 r+1)^{p-j+1}}\right] \\
& +\sum_{j=1}^{p}\left|A_{j}\right| \sum_{n=1}^{\infty}\left[\frac{(-1)^{j+1}}{(2 n-1)^{p-j+1}}+\frac{(-1)^{p}}{(2 n-1)^{p-j+1}}\right] .
\end{aligned}
$$

The second term in the last expression can be simplified until we obtain

$$
\begin{aligned}
\sum_{n=1}^{\infty} \frac{1}{\left(4 n^{2}-(2 a+1)^{2}\right)^{p}}=\sum_{j=1}^{p} & \frac{(-1)^{p+1}\left|A_{j}\right|}{(2 a+1)^{p-j+1}}+\sum_{j=1}^{p}\left|A_{j}\right| \sum_{r=1}^{a}\left[\frac{(-1)^{p+1}-(-1)^{p+1}}{(2 r-1)^{p-j+1}}\right] \\
& +\sum_{j=1}^{p}\left|A_{j}\right| \sum_{n=1}^{\infty}\left[\frac{(-1)^{j+1}}{(2 n-1)^{p-j+1}}+\frac{(-1)^{p}}{(2 n-1)^{p-j+1}}\right] .
\end{aligned}
$$


TABLE 2.1. Some values of $\zeta(p)$ and $S(a, p)$.

\begin{tabular}{l|l|l}
\hline$p$ & $\zeta(p)$ & $S(a, p)$ \\
\hline 1 & - & $\frac{1}{2(2 a+1)^{2}}$ \\
\hline 2 & $\frac{\pi^{2}}{6}$ & $\frac{\pi^{2}}{16(2 a+1)^{2}}-\frac{1}{2(2 a+1)^{4}}$ \\
\hline 3 & - & $\frac{1}{2(2 a+1)^{6}}-\frac{3 \pi^{2}}{64(2 a+1)^{6}}$ \\
\hline 4 & $\frac{\pi^{4}}{90}$ & $\frac{\pi^{4}}{768(2 a+1)^{4}}+\frac{5 \pi^{2}}{128(2 a+1)^{6}}-\frac{1}{2(2 a+1)^{8}}$ \\
\hline 5 & - & $\frac{1}{2(2 a+1)^{10}}-\frac{35 \pi^{2}}{1024(2 a+1)^{8}}-\frac{5 \pi^{4}}{3072(2 a+1)^{6}}$ \\
\hline 6 & $\frac{\pi^{6}}{945}$ & $\frac{\pi^{6}}{30720(2 a+1)^{6}}+\frac{7 \pi^{4}}{4096(2 a+1)^{8}}+\frac{63 \pi^{2}}{2048(2 a+1)^{10}}-\frac{1}{2(2 a+1)^{12}}$ \\
\hline 7 & - & $\frac{1}{2(2 a+1)^{14}}-\frac{231 \pi^{2}}{8192(2 a+1)^{12}}-\frac{7 \pi^{4}}{4096(2 a+1)^{10}}-\frac{122880(2 a+1)^{8}}{1229 \pi^{6}}$ \\
\hline 8 & $\frac{\pi^{8}}{9450}$ & $\frac{17 \pi^{8}}{2^{16} \cdot 3^{2} \cdot 5 \cdot 7(2 a+1)^{8}}+\frac{3 \pi^{13} \cdot 5 \cdot(2 a+1)^{10}}{2^{10}} \frac{55 \pi^{4}}{2^{15} \cdot(2 a+1)^{12}}+\frac{429 \pi^{2}}{2^{11} \cdot(2 a+1)^{14}}-\frac{1}{2(2 a+1)^{16}}$ \\
\hline 9 & - & $\frac{1}{2(2 a+1)^{18}}-\frac{6435 \pi^{2}}{2^{18}(2 a+1)^{16}}-\frac{429 \pi^{4}}{2^{18} \cdot(2 a+1)^{14}}-\frac{11 \pi^{6}}{2^{17} \cdot(2 a+1)^{12}}-\frac{17 \pi^{8}}{2^{18} \cdot 35(2 a+1)^{10}}$ \\
\hline
\end{tabular}

Notice that on the right-hand side we have the last term

$$
T(p, j)=\sum_{j=1}^{p}\left|A_{j}\right| \sum_{n=1}^{\infty}\left[\frac{(-1)^{j+1}}{(2 n-1)^{p-j+1}}+\frac{(-1)^{p}}{(2 n-1)^{p-j+1}}\right] .
$$

For $j=p$, we have

$$
T(p, p)=\left|A_{p}\right| \sum_{n=1}^{\infty}\left(\frac{(-1)^{p}}{2 n-1}-\frac{(-1)^{p}}{2 n-1}\right)=0,
$$

which causes the annihilation of any possible contribution from a divergent series.

Now if we use Lemma 2.2 and (2.1), we obtain

$$
S(a, p)=\frac{(-1)^{p+1}}{2(2 a+1)^{2 p}}+\sum_{j=1}^{p}\left|A_{j}\right|\left((-1)^{j+1}+(-1)^{p}\right)\left(1-\frac{1}{2^{p-j+1}}\right) \zeta(p-j+1),
$$

and by the change of counter $k=p-j+1$ we arrive at our result (2.21), hence the theorem is proved.

Some values of $\zeta(p)$ and $S(a, p)$ are listed in Table 2.1.

Jolley [7] lists the values of $S(0,1), S(0,2)$, and $S(0,3)$, which he attributes to Adams [2]. Jolley also lists

$$
\sum_{n=1}^{\infty} \frac{n}{\left(4 n^{2}-1\right)^{2}}=\frac{1}{8}
$$


moreover using (2.31) and (2.3) results in

$$
\begin{aligned}
S(a, 2) & =\sum_{n=1}^{\infty} \frac{n}{\left(4 n^{2}-(2 a+1)^{2}\right)^{2}}=\frac{1}{8(2 a+1)} \sum_{r=1}^{2 a+1} \frac{1}{(2 r-(2 a+1))^{2}} \\
& =\frac{1}{8(2 a+1)^{3}}+\frac{1}{4(2 a+1)} \sum_{r=1}^{a} \frac{1}{(2 a+1-2 r)^{2}},
\end{aligned}
$$

and putting $a=0$ results in (2.31).

From (2.3) and for $p=3$,

$$
\sum_{n=1}^{\infty} \frac{n^{2}}{\left(4 n^{2}-(2 a+1)^{2}\right)^{3}}=\frac{\pi^{2}}{256(2 a+1)^{2}},
$$

and for $p=5$, we have

$$
\sum_{n=1}^{\infty} \frac{2 n^{4}+n^{2}(2 a+1)^{2}}{\left(4 n^{2}-(2 a+1)^{2}\right)^{5}}=\frac{\pi^{4}}{2^{13} \cdot 3 \cdot(2 a+1)^{2}}+\frac{7 \pi^{2}}{2^{13} \cdot(2 a+1)^{4}} .
$$

We now deal with the alternating case.

3. Quadratic alternating case. We first state the following lemma which will be useful later.

LEMMA 3.1. For $p=1,2,3, \ldots$ and $a$ an integer bigger than or equal to zero, (i)

$$
\sum_{n=1}^{\infty} \frac{(-1)^{n+1}}{(2 n-(2 a+1))^{p}}=\sum_{r=1}^{a} \frac{(-1)^{r+1}}{(2 r-(2 a+1))^{p}}+(-1)^{a} \sigma(p),
$$

(ii)

$$
\sum_{n=1}^{\infty} \frac{(-1)^{n+1}}{(2 n+2 a+1)^{p}}=\frac{1}{(2 a+1)^{p}}-(-1)^{a} \sum_{r=1}^{a} \frac{(-1)^{r}}{(2 r-1)^{p}}-(-1)^{a} \sigma(p),
$$

(iii)

$$
\begin{aligned}
& \sum_{n=1}^{\infty}(-1)^{n+1}\left\{\frac{(2 n+(2 a+1))^{p}+(2 n-(2 a+1))^{p}}{\left(4 n^{2}-(2 a+1)^{2}\right)^{p}}\right\} \\
& \quad=\sum_{r=1}^{2 a+1} \frac{(-1)^{r+1}}{(2 r-(2 a+1))^{p}}=\frac{1}{(2 a+1)^{p}}+ \begin{cases}0, & \text { for } p \text { even, } \\
\sum_{r=1}^{a} \frac{2(-1)^{r}}{(2 a+1-2 r)^{p}}, & \text { for } p \text { odd } .\end{cases}
\end{aligned}
$$


Proof. (i) We have

$$
\begin{aligned}
& \sum_{n=1}^{\infty} \frac{(-1)^{n+1}}{(2 n-(2 a+1))^{p}} \\
& \quad=\frac{1}{(1-2 a)^{p}}-\frac{1}{(3-2 a)^{p}}+\cdots+\frac{(-1)^{a+1}}{(-1)^{p}}+\frac{(-1)^{a+2}}{1^{p}}+\frac{(-1)^{a+3}}{3^{p}}+\frac{(-1)^{a+4}}{5^{p}}+\cdots \\
& =\sum_{r=1}^{a} \frac{(-1)^{r+1}}{(2 r-1-2 a)^{p}}+\sum_{n=1}^{\infty} \frac{(-1)^{a+n+1}}{(2 n-1)^{p}} \\
& =\sum_{r=1}^{a} \frac{(-1)^{r+1}}{(2 r-(2 a+1))^{p}}+(-1)^{a} \sigma(p) .
\end{aligned}
$$

(ii) Firstly we have

$$
\sum_{n=1}^{\infty} \frac{(-1)^{n+1}}{(2 n+2 a+1)^{p}}=\sum_{r=1}^{2 a+1} \frac{(-1)^{r+1}}{((2 r-1)-2 a)^{p}}-\sum_{n=1}^{\infty} \frac{(-1)^{n+1}}{(2 n-(2 a+1))^{p}} .
$$

From part (i) we can write

$$
\begin{aligned}
\sum_{n=1}^{\infty} \frac{(-1)^{n+1}}{(2 n+2 a+1)^{p}}= & \sum_{r=1}^{a} \frac{(-1)^{r+1}}{((2 r-1)-2 a)^{p}}+\sum_{r=a+1}^{2 a+1} \frac{(-1)^{r+1}}{((2 r-1)-2 a)^{p}} \\
& -\sum_{r=1}^{a} \frac{(-1)^{r+1}}{(2 r-1-2 a)^{p}}-(-1)^{a} \sum_{n=1}^{\infty} \frac{(-1)^{n+1}}{(2 n-1)^{p}}
\end{aligned}
$$

and changing the counter in the second term we have

$$
\begin{aligned}
\sum_{n=1}^{\infty} \frac{(-1)^{n+1}}{(2 n+2 a+1)^{p}} & =\sum_{r=0}^{a} \frac{(-1)^{r+a}}{(2 r+1)^{p}}-(-1)^{a} \sum_{n=1}^{\infty} \frac{(-1)^{n+1}}{(2 n-1)^{p}} \\
& =\frac{1}{(2 a+1)^{p}}-(-1)^{a} \sum_{r=1}^{a} \frac{(-1)^{r}}{(2 r-1)^{p}}-(-1)^{a} \sigma(p) .
\end{aligned}
$$

(iii) We have

$$
\begin{aligned}
Q:= & \sum_{n=1}^{\infty}\left[\frac{(-1)^{n+1}}{(2 n-(2 a+1))^{p}}+\frac{(-1)^{n+1}}{(2 n+(2 a+1))^{p}}\right] \\
= & \sum_{n=1}^{\infty}(-1)^{n+1}\left[\frac{(2 n+(2 a+1))^{p}+(2 n-(2 a+1))^{p}}{\left(4 n^{2}-(2 a+1)^{2}\right)^{p}}\right] \\
= & \sum_{r=1}^{a} \frac{(-1)^{r+1}}{(2 r-2 a-1)^{p}}+(-1)^{a} \sum_{n=1}^{\infty} \frac{(-1)^{n+1}}{(2 n-1)^{p}} \\
& \quad-(-1)^{a} \sum_{n=1}^{\infty} \frac{(-1)^{n+1}}{(2 n-1)^{p}}-(-1)^{a} \sum_{r=1}^{a+1} \frac{(-1)^{r}}{(2 r-1)^{p}}
\end{aligned}
$$

from (i) and (ii). 
Now

$$
\begin{aligned}
Q & =\sum_{r=1}^{2 a+1} \frac{(-1)^{r+1}}{(2 r-2 a-1)^{p}} \\
& =\frac{1}{(2 a+1)^{p}}+\sum_{r=1}^{a} \frac{(-1)^{r+1}}{(2 r-2 a-1)^{p}}+(-1)^{a} \sum_{r=1}^{a} \frac{(-1)^{r+1}}{(2 r-1)^{p}} \\
& =\frac{1}{(2 a+1)^{p}}+\sum_{r=1}^{a} \frac{(-1)^{r+1}\left((-1)^{p}-1\right)}{(2 a+1-2 r)^{p}} \\
& =\frac{1}{(2 a+1)^{p}}+ \begin{cases}0, & \text { for } p \text { even, } \\
\sum_{r=1}^{a} \frac{2(-1)^{r}}{(2 a+1-2 r)^{p}}, & \text { for } p \text { odd. }\end{cases}
\end{aligned}
$$

The main theorem is now proved.

THEOREM 3.2. For $p=1,2,3 \ldots$ and $a \in \mathbb{N} \cup\{0\}$,

$$
A S(a, p)=\frac{(-1)^{p}}{2(2 a+1)^{2 p}}-(-1)^{p+a} \sum_{k=1}^{p}\left|A_{p-k+1}\right|\left(1-(-1)^{k}\right) \sigma(k),
$$

where $A_{j}$ is defined by (2.16) and $\sigma(p)$ by (1.6).

Proof. We have

$$
\sum_{n=1}^{\infty} \frac{(-1)^{n+1}}{\left(4 n^{2}-(2 a+1)^{2}\right)^{p}}=\sum_{n=1}^{\infty}(-1)^{n+1} \sum_{j=1}^{p}\left[\frac{A_{j}}{(2 n-2 a-1)^{p-j+1}}+\frac{B_{j}}{(2 n+2 a+1)^{p-j+1}}\right],
$$

where $A_{j}$ is defined by (2.16) and $B_{j}$ by (2.23). $A_{j}$ and $B_{j}$ are related by (2.24) and hence we can write

$$
\sum_{n=1}^{\infty} \frac{(-1)^{n+1}}{\left(4 n^{2}-(2 a+1)^{2}\right)^{p}}=\sum_{n=1}^{\infty}(-1)^{n+1} \sum_{j=1}^{p}\left[\frac{(-1)^{j+1}\left|A_{j}\right|}{(2 n-2 a-1)^{p-j+1}}+\frac{(-1)^{p}\left|A_{j}\right|}{(2 n+2 a+1)^{p-j+1}}\right] .
$$

Interchanging sums gives us

$$
\sum_{n=1}^{\infty} \frac{(-1)^{n+1}}{\left(4 n^{2}-(2 a+1)^{2}\right)^{p}}=\sum_{j=1}^{p}\left|A_{j}\right| \sum_{n=1}^{\infty}(-1)^{n+1}\left[\frac{(-1)^{j+1}}{(2 n-2 a-1)^{p-j+1}}+\frac{(-1)^{p}}{(2 n+2 a+1)^{p-j+1}}\right] .
$$


Utilising (3.1) and (3.2), we have

$$
\begin{aligned}
\sum_{n=1}^{\infty} & \frac{(-1)^{n+1}}{\left(4 n^{2}-(2 a+1)^{2}\right)^{p}} \\
= & \sum_{j=1}^{p}\left|A_{j}\right|\left[(-1)^{j+a+1} \sum_{n=1}^{\infty} \frac{(-1)^{n+1}}{(2 n-1)^{p-j+1}}+(-1)^{j+1} \sum_{r=1}^{a} \frac{(-1)^{r+1}}{(2 r-2 a-1)^{p-j+1}}\right. \\
& \left.\quad+\frac{(-1)^{p}}{(2 a+1)^{p-j+1}}+(-1)^{p+a} \sum_{r=1}^{a} \frac{(-1)^{r+1}}{(2 r-1)^{p-j+1}}-(-1)^{p+a} \sum_{n=1}^{\infty} \frac{(-1)^{n+1}}{(2 n-1)^{p-j+1}}\right] \\
= & \sum_{j=1}^{p} \frac{(-1)^{p}\left|A_{j}\right|}{(2 a+1)^{p-j+1}} \\
& +\sum_{j=1}^{p}\left|A_{j}\right|\left[(-1)^{j+1} \sum_{r=1}^{a} \frac{(-1)^{r+1}}{(2 r-2 a-1)^{p-j+1}}+(-1)^{p+a} \sum_{r=1}^{a} \frac{(-1)^{r+1}}{(2 r-1)^{p-j+1}}\right] \\
& +\sum_{j=1}^{p}\left|A_{j}\right|\left[(-1)^{j+1+a} \sum_{n=1}^{\infty} \frac{(-1)^{n+1}}{(2 n-1)^{p-j+1}}-(-1)^{p+a} \sum_{n=1}^{\infty} \frac{(-1)^{n+1}}{(2 n-1)^{p-j+1}}\right] \\
= & \sum_{j=1}^{p} \frac{(-1)^{p}\left|A_{j}\right|}{(2 a+1)^{p-j+1}}+\sum_{j=1}^{p}\left|A_{j}\right|\left((-1)^{j+1+a}+(-1)^{p+1+a}\right) \sum_{n=1}^{\infty} \frac{(-1)^{n+1}}{(2 n-1)^{p-j+1}} \\
& +\sum_{j=1}^{p}\left|A_{j}\right|\left[\sum_{r=1}^{a} \frac{(-1)^{r+j}}{(2 r-2 a-1)^{p-j+1}}-\sum_{r=1}^{a} \frac{(-1)^{p+a+r}}{(2 r-1)^{p-j+1}}\right] .
\end{aligned}
$$

The inside sums of the last term in (3.14) can be simplified as follows:

$$
\sum_{r=1}^{a}\left[\frac{(-1)^{r+j}}{(2 r-2 a-1)^{p-j+1}}-\frac{(-1)^{p+a+r}}{(2 r-1)^{p-j+1}}\right]=\sum_{r=1}^{a}\left[\frac{(-1)^{r+p+1}}{(2 a+1-2 r)^{p-j+1}}-\frac{(-1)^{p+a+r}}{(2 r-1)^{p-j+1}}\right] .
$$

Collecting first and last terms, second and second last terms, and so forth, we have from (3.15)

$$
\begin{aligned}
& \sum_{r=1}^{a}\left[\frac{(-1)^{r+p+1}}{(2 a+1-2 r)^{p-j+1}}-\frac{(-1)^{p+a+r}}{(2 r-1)^{p-j+1}}\right] \\
& =\sum_{r=1}^{a}\left[\frac{(-1)^{p+1+r}}{(2 a+1-2 r)^{p-j+1}}-\frac{(-1)^{p+a+a+1-r}}{(2 a+1-2 r)^{p-j+1}}\right]=0 .
\end{aligned}
$$

Hence, from (3.14),

$$
\begin{aligned}
& \sum_{n=1}^{\infty} \frac{(-1)^{n+1}}{\left(4 n^{2}-(2 a+1)^{2}\right)^{p}} \\
& \quad=\sum_{j=1}^{p} \frac{(-1)^{p}\left|A_{j}\right|}{(2 a+1)^{p-j+1}}-(-1)^{a} \sum_{j=1}^{p}\left|A_{j}\right|\left((-1)^{j}+(-1)^{p}\right) \sum_{n=1}^{\infty} \frac{(-1)^{n+1}}{(2 n-1)^{p-j+1}} .
\end{aligned}
$$


TABLE 3.1. Some values of $\sigma(p)$ and $A S(p)$.

\begin{tabular}{l|l|l}
\hline$p$ & $\sigma(p)$ & $A S(p)$ \\
\hline 1 & $\frac{\pi}{4}$ & $\frac{(-1)^{a} \pi}{4(2 a+1)}-\frac{1}{2(2 a+1)^{2}}$ \\
\hline 2 & - & $\frac{1}{2(2 a+1)^{4}}-\frac{(-1)^{a} \pi}{8(2 a+1)^{3}}$ \\
\hline 3 & $\frac{\pi^{3}}{32}$ & $\frac{(-1)^{a} \pi^{3}}{128(2 a+1)^{3}}+\frac{(-1)^{a} \cdot 3 \cdot \pi}{32(2 a+1)^{5}}-\frac{1}{2(2 a+1)^{6}}$ \\
\hline 4 & - & $\frac{1}{2(2 a+1)^{8}}-\frac{(-1)^{a} \cdot 5 \cdot \pi}{64(2 a+1)^{7}}-\frac{(-1)^{a} \pi^{3}}{128(2 a+1)^{5}}$ \\
\hline 5 & $\frac{5 \pi^{5}}{1536}$ & $\frac{(-1)^{a} \cdot 5 \cdot \pi^{5}}{2^{13} \cdot 3 \cdot(2 a+1)^{5}}+\frac{(-1)^{a} \cdot 15 \cdot \pi^{3}}{2^{11} \cdot(2 a+1)^{7}}+\frac{(-1)^{a} \cdot 35 \cdot \pi}{2^{9}(2 a+1)^{9}}-\frac{1}{2(2 a+1)^{10}}$ \\
\hline 6 & - & $\frac{1}{2(2 a+1)^{12}}-\frac{(-1)^{a} \cdot 63 \cdot \pi}{2^{10} \cdot(2 a+1)^{11}}-\frac{(-1)^{a} \cdot 7 \cdot \pi^{3}}{2^{10} \cdot(2 a+1)^{9}}-\frac{(-1)^{a} \cdot 5 \cdot \pi^{5}}{2^{14} \cdot(2 a+1)^{7}}$ \\
\hline 7 & $\frac{61 \pi^{7}}{184320}$ & $\frac{(-1)^{a} \cdot 61 \cdot \pi^{7}}{2^{18} \cdot 3^{2} \cdot 5 \cdot(2 a+1)^{7}}+\frac{(-1)^{a} \cdot 35 \cdot \pi^{5}}{2^{15} \cdot(2 a+1)^{9}}+\frac{(-1)^{a} \cdot 105 \cdot \pi^{3}}{2^{14} \cdot(2 a+1)^{11}}+\frac{(-1)^{a} \cdot 231 \cdot \pi}{2^{12} \cdot(2 a+1)^{13}}-\frac{1}{2(2 a+1)^{14}}$ \\
\hline 8 & - & $\frac{1}{2(2 a+1)^{16}}-\frac{(-1)^{a} \cdot 429 \cdot \pi}{2^{13} \cdot(2 a+1)^{15}}-\frac{(-1)^{a} \cdot 99 \cdot \pi^{3}}{2^{14} \cdot(2 a+1)^{13}}-\frac{(-1)^{a} \cdot 25 \cdot \pi^{5}}{2^{16} \cdot(2 a+1)^{11}}-\frac{(-1)^{a} \cdot 61 \cdot \pi^{7}}{2^{17} \cdot 3^{2} \cdot 5 \cdot(2 a+1)^{9}}$ \\
\hline
\end{tabular}

Now using Lemma 2.2, we have

$$
A S(a, p)=\frac{(-1)^{p}}{2(2 a+1)^{2 p}}-(-1)^{a} \sum_{j=1}^{p}\left|A_{j}\right|\left((-1)^{j}+(-1)^{p}\right) \sigma(p-j+1),
$$

and by the change of counter $k=p-j+1$, we arrive at our result (3.10), hence the theorem is proved.

Table 3.1 lists some values of $\sigma(p)$ and $A S(p)$.

Jolley [7] lists the value of $A S(0,1)$ and some particular cases of $A S(a, p)$ are also given by Gradshteyn and Ryzhik [6].

From Lemma 3.1, for $p=2$ we get

$$
\sum_{n=1}^{\infty} \frac{(-1)^{n+1}\left(8 n^{2}+2(2 a+1)^{2}\right)}{\left(4 n^{2}-(2 a+1)^{2}\right)^{2}}=\frac{1}{(2 a+1)^{2}} .
$$

From Theorem 3.2, where for $p=2$

$$
\left|A_{2}\right|=\frac{1}{4(2 a+1)^{3}},
$$

we have

$$
\sum_{n=1}^{\infty} \frac{(-1)^{n+1}}{\left(4 n^{2}-(2 a+1)^{2}\right)^{2}}=\frac{1}{2(2 a+1)^{2}}-\frac{(-1)^{a} \pi}{8(2 a+1)^{3}},
$$

and therefore

$$
\sum_{n=1}^{\infty} \frac{(-1)^{n+1} n^{2}}{\left(4 n^{2}-(2 a+1)^{2}\right)^{2}}=\frac{(-1)^{a} \pi}{32(2 a+1)} .
$$


For $p=4$,

$$
\sum_{n=1}^{\infty} \frac{(-1)^{n+1}\left(2 n^{4}+3 n^{2}(2 a+1)^{2}\right)}{\left(4 n^{2}-(2 a+1)^{2}\right)^{4}}=\frac{(-1)^{a} 5 \pi}{2^{9} \cdot(2 a+1)^{3}}+\frac{(-1)^{a} \pi^{3}}{2^{10} \cdot(2 a+1)},
$$

and for $p=3$,

$$
\sum_{n=1}^{\infty}(-1)^{n+1} \frac{\left(4 n^{3}+3 n(2 a+1)^{2}\right)}{\left(4 n^{2}-(2 a+1)^{2}\right)^{3}}=\frac{1}{4(2 a+1)^{3}}+\frac{1}{2} \sum_{r=1}^{a} \frac{(-1)^{r}}{(2 a+1-2 r)^{3}}
$$

4. The hypergeometric expression. The series (1.1), (1.5), and (1.9) can be represented as a generalised hypergeometric function, see, for example, [5]. Consider the series (1.5),

$$
\sum_{n=0}^{\infty} \frac{(-1)^{n+1}}{\left(4 n^{2}-(2 a+1)^{2}\right)^{p}}=\frac{(-1)^{p+1}}{(2 a+1)^{2 p}}+\sum_{n=1}^{\infty} \frac{(-1)^{n+1}}{\left(4 n^{2}-(2 a+1)^{2}\right)^{p}},
$$

let $T_{n}=(-1)^{n+1} /\left(4 n^{2}-(2 a+1)^{2}\right)^{p}$ where $T_{0}=(-1)^{p+1} /(2 a+1)^{2 p}$, hence

$$
\frac{T_{n+1}}{T_{n}}=-\frac{(n+1)(n+1 / 2+a)^{p}(n-1 / 2-a)^{p}}{(n+1)(n+3 / 2+a)^{p}(n+1 / 2-a)^{p}} .
$$

We can now write

$$
\begin{aligned}
\sum_{n=1}^{\infty} \frac{(-1)^{n+1}}{\left(4 n^{2}-(2 a+1)^{2}\right)^{p}}=\frac{(-1)^{p}}{(2 a+1)^{2 p}} & \\
& +T_{02 p+1} F_{2 p}\left[\begin{array}{c}
1, \frac{1}{2}+a, \frac{1}{2}+a, \ldots, \frac{1}{2}+a,-\frac{1}{2}-a,-\frac{1}{2}-a, \ldots,-\frac{1}{2}-a \mid-1 \\
\frac{3}{2}+a, \frac{3}{2}+a, \ldots, \frac{3}{2}+a, \frac{1}{2}-a, \frac{1}{2}-a, \ldots, \frac{1}{2}-a
\end{array}\right],
\end{aligned}
$$

and from (3.10),

$$
\begin{gathered}
2 p+1 F_{2 p}\left[\begin{array}{c}
1, \frac{1}{2}+a, \frac{1}{2}+a, \ldots, \frac{1}{2}+a,-\frac{1}{2}-a,-\frac{1}{2}-a, \ldots,-\frac{1}{2}-a \\
\frac{3}{2}+a, \frac{3}{2}+a, \ldots, \frac{3}{2}+a, \frac{1}{2}-a, \frac{1}{2}-a, \ldots, \frac{1}{2}-a
\end{array} \mid-1\right] \\
=\frac{1}{2}+(-1)^{a}(2 a+1)^{2 p} \sum_{k=1}^{p}\left|A_{p-k+1}\right|\left(1-(-1)^{k}\right) \sigma(k),
\end{gathered}
$$

where $A_{j}$ is defined by (2.16). 
REMARK 4.1. The series (1.1) and (1.4) can be expressed in terms of the Lerch transcedent or the Catalan beta function. In particular, from (1.4)

$$
\sum_{n=1}^{\infty} \frac{(-1)^{n+1}}{\left(4 n^{2}-(2 a+1)^{2}\right)^{p}}=\frac{(-1)^{p}}{(2 a+1)^{2 p}}+\sum_{n=0}^{\infty} \frac{(-1)^{n+1}}{\left(4 n^{2}-(2 a+1)^{2}\right)^{p}}
$$

The Lerch transcedent, $\Phi(z, s, \alpha)$ is defined as

$$
\Phi(z, s, \alpha)=\sum_{n=0}^{\infty} \frac{z^{n}}{(n+\alpha)^{s}}
$$

where the $n+\alpha=0$ term is excluded from the sum.

The Catalan beta function, $\beta(s)$, is as follows:

$$
\beta(s)=\sum_{n=0}^{\infty} \frac{(-1)^{n}}{(2 n+1)^{s}}=2^{-s} \Phi\left(-1, s, \frac{1}{2}\right)
$$

and in particular the Catalan constant, for $s=2$ is

$$
\beta(2)=C=\sum_{n=0}^{\infty} \frac{(-1)^{n}}{(2 n+1)^{2}}=\frac{1}{4} \Phi\left(-1,2, \frac{1}{2}\right) \sim 0.91596
$$

Moreover, we note that the generalised Zeta function $\zeta(s, a)$ is defined by

$$
\begin{gathered}
\zeta(s, a):=\Phi(1, s, \alpha)=\sum_{n=0}^{\infty} \frac{1}{(n+\alpha)^{s}}, \\
\mathbb{R}(s)>1, \quad \alpha \in \mathbb{C} \mid \mathbb{Z}_{0}^{-} ; \quad \mathbb{Z}_{0}^{-}:=\mathbb{Z}^{-} \cup\{0\} ; \quad \mathbb{Z}^{-}:=\{-1,-2,-3, \ldots\} .
\end{gathered}
$$

For a definition of the generalised Zeta function and closed form representation of series involving Zeta functions the reader is referred to the excellent article by Lee and Choi [8].

The polygamma functions $\Psi^{(k)}(z), k \in \mathbb{N}$, are defined by

$$
\Psi^{(k)}(z):=\frac{d^{k+1}}{d z^{k+1}} \log \Gamma(z)=\frac{d^{k}}{d z^{k}} \Psi(z) \quad k \in \mathbb{N}_{0}:=\mathbb{N} \cup\{0\}
$$

where $\Psi^{(0)}(z):=\Psi(z)$ denotes the Psi (or digamma) function defined by

$$
\Psi(z):=\frac{d}{d z} \log \Gamma(z)=\frac{\Gamma^{\prime}(z)}{\Gamma(z)} \quad \text { or } \quad \log \Gamma(z)=\int_{1}^{z} \Psi(t) d t
$$

In terms of the generalised Zeta function we may write

$$
\Psi^{(k)}(z)=(-1)^{k+1} k ! \zeta(k+1, z) .
$$


From (4.5), (4.6), and (4.7)

$$
\begin{aligned}
& \sum_{n=1}^{\infty} \frac{(-1)^{n+1}}{\left(4 n^{2}-(2 a+1)^{2}\right)^{p}} \\
&=\frac{(-1)^{p}}{(2 a+1)^{2 p}}+\sum_{n=0}^{\infty}(-1)^{n+1} \sum_{j=1}^{p}\left[\frac{A_{j}}{(2 n-2 a-1)^{p-j+1}}+\frac{B_{j}}{(2 n+2 a+1)^{p-j+1}}\right] \\
&=\frac{(-1)^{p}}{(2 a+1)^{2 p}}-\sum_{j=1}^{p}\left[\sum_{n=0}^{\infty} \frac{(-1)^{n} A_{j}}{(2 n-2 a-1)^{p-j+1}}+\frac{(-1)^{n} B_{j}}{(2 n+2 a+1)^{p-j+1}}\right] \\
&=\frac{(-1)^{p}}{(2 a+1)^{2 p}}-\sum_{j=1}^{p} 2^{-(p-j+1)}\left[A_{j} \Phi\left(-1, p-j+1,-a-\frac{1}{2}\right)+B_{j} \Phi\left(-1, p-j+1, a+\frac{1}{2}\right)\right] \\
&=\frac{(-1)^{p}}{(2 a+1)^{2 p}}-\sum_{j=1}^{p}\left[2^{-(p-j+1)} A_{j} \Phi\left(-1, p-j+1,-a-\frac{1}{2}\right)\right. \\
&\left.\quad+B_{j}\left(\frac{1}{(2 a+1)^{p-j+1}}+(-1)^{a} \sum_{r=0}^{a} \frac{(-1)^{r}}{(2 r+1)^{p-j+1}}-(-1)^{a} \beta(p-j+1)\right)\right] .
\end{aligned}
$$

From the relationship between $A_{j}$ and $B_{j}$ we have

$$
\begin{aligned}
& \sum_{n=1}^{\infty} \frac{(-1)^{n+1}}{\left(4 n^{2}-(2 a+1)^{2}\right)^{p}} \\
& =\frac{(-1)^{p}}{(2 a+1)^{2 p}}-\sum_{j=1}^{p}\left|A_{j}\right| 2^{-(p-j+1)}\left[(-1)^{j+1} \Phi\left(-1, p-j+1,-a-\frac{1}{2}\right)\right. \\
& \left.+(-1)^{p} \Phi\left(-1, p-j+1, a+\frac{1}{2}\right)\right],
\end{aligned}
$$

and by the change of counter $k=p-j+1$, we have

$$
\begin{aligned}
& \sum_{n=1}^{\infty} \frac{(-1)^{n+1}}{\left(4 n^{2}-(2 a+1)^{2}\right)^{p}} \\
& \quad=\frac{(-1)^{p}}{(2 a+1)^{2 p}}-(-1)^{p} \sum_{k=1}^{p}\left|A_{p-k+1}\right| 2^{-k}\left[(-1)^{k} \Phi\left(-1, k,-a-\frac{1}{2}\right)+\Phi\left(-1, k, a+\frac{1}{2}\right)\right] .
\end{aligned}
$$

From (3.10), (4.3), (4.4), and (4.15) we have

$$
\begin{aligned}
T_{02 p+1} F_{2 p}\left[\begin{array}{c}
1, \frac{1}{2}+a, \frac{1}{2}+a, \ldots, \frac{1}{2}+a,-\frac{1}{2}-a,-\frac{1}{2}-a, \ldots,-\frac{1}{2}-a \mid-1 \\
\frac{3}{2}+a, \frac{3}{2}+a, \ldots, \frac{3}{2}+a, \frac{1}{2}-a, \frac{1}{2}-a, \ldots, \frac{1}{2}-a
\end{array}\right] \\
=(-1)^{p} \sum_{k=1}^{p}\left|A_{p-k+1}\right| 2^{-k}\left[(-1)^{k} \Phi\left(-1, k,-a-\frac{1}{2}\right)+\Phi\left(-1, k, a+\frac{1}{2}\right)\right] \\
=\frac{(-1)^{p+1}}{2(2 a+1)^{2 p}}-(-1)^{p+a} \sum_{k=1}^{p}\left|A_{p-k+1}\right|\left(1-(-1)^{k}\right) \sigma(k) .
\end{aligned}
$$


Since we can write

$$
\begin{aligned}
\sigma(k) & =\sum_{n=1}^{\infty} \frac{(-1)^{n+1}}{(2 n-1)^{k}}=\sum_{n=1}^{\infty} \frac{1}{(2 n-1)^{k}}-\sum_{n=1}^{\infty} \frac{2}{(4 n-1)^{k}} \\
& =\delta(k)-2^{1-2 k} \sum_{n=0}^{\infty} \frac{1}{(n-1 / 4)^{k}}+2(-1)^{k} \\
& =\left(1-\frac{1}{2^{k}}\right) \sum_{n=1}^{\infty} \frac{1}{n^{k}}-2^{1-2 k} \Phi\left(1, k,-\frac{1}{4}\right)+2(-1)^{k} \\
& =\left(1-\frac{1}{2^{k}}\right) \zeta(k)-2^{1-2 k} \Phi\left(1, k,-\frac{1}{4}\right)+2(-1)^{k}, \\
\sigma(k) & =\left(1-\frac{1}{2^{k}}\right) \zeta(k)-2^{1-2 k} \zeta\left(k,-\frac{1}{4}\right)+2(-1)^{k} \\
& =\left(1-\frac{1}{2^{k}}\right) \zeta(k)-2^{1-2 k} \frac{(-1)^{k} \Psi^{(k-1)}(-1 / 4)}{(k-1) !}+2(-1)^{k},
\end{aligned}
$$

we now have

$$
\begin{aligned}
T_{02 p+1} F_{2 p}\left[\begin{array}{c}
1, \\
\frac{1}{2}+a, \frac{1}{2}+a, \ldots, \frac{1}{2}+a,-\frac{1}{2}-a,-\frac{1}{2}-a, \ldots,-\frac{1}{2}-a \mid-1 \\
\frac{3}{2}+a, \frac{3}{2}+a, \ldots, \frac{3}{2}+a, \frac{1}{2}-a, \frac{1}{2}-a, \ldots, \frac{1}{2}-a
\end{array}\right] \\
=\frac{(-1)^{p+1}}{2(2 a+1)^{2 p}}-(-1)^{p+a} \sum_{k=1}^{p}\left|A_{p-k+1}\right|\left(1-(-1)^{k}\right) \\
\quad \times\left[\left(1-\frac{1}{2^{k}}\right) \zeta(k)-2^{1-2 k} \Phi\left(1, k,-\frac{1}{4}\right)+2(-1)^{k}\right]
\end{aligned}
$$

and from (3.10), we have

$$
\begin{aligned}
& A S(a, p)= \frac{(-1)^{p}}{2(2 a+1)^{2 p}}-(-1)^{p+a} \sum_{k=1}^{p}\left|A_{p-k+1}\right|\left(1-(-1)^{k}\right) \\
& \quad \times\left[\left(1-\frac{1}{2^{k}}\right) \zeta(k)-2^{1-2 k} \Phi\left(1, k,-\frac{1}{4}\right)+2(-1)^{k}\right], \\
& A S(a, p)=\sum_{n=1}^{\infty} \frac{(-1)^{n+1}}{\left(4 n^{2}-(2 a+1)^{2}\right)^{p}} \\
&=\frac{(-1)^{p}}{2(2 a+1)^{2 p}}-(-1)^{p+a} \sum_{k=1}^{p}\left|A_{p-k+1}\right|\left(1-(-1)^{k}\right) \\
& \quad \times\left[\left(1-\frac{1}{2^{k}}\right) \zeta(k)-2^{1-2 k} \zeta\left(k,-\frac{1}{4}\right)+2(-1)^{k}\right] \\
&=\frac{(-1)^{p}}{2(2 a+1)^{2 p}}-(-1)^{p+a} \sum_{k=1}^{p}\left|A_{p-k+1}\right|\left(1-(-1)^{k}\right) \\
& \quad \times\left[\left(1-\frac{1}{2^{k}}\right) \zeta(k)-\frac{2^{1-2 k}(-1)^{k} \Psi^{(k-1)}(-1 / 4)}{(k-1) !}+2(-1)^{k}\right] .
\end{aligned}
$$

Various particular values of $A S(a, p)$ are also given by Abramowitz and Stegun [1]. 
From (3.1) and (3.2) we can subtract the quantities to obtain

$$
\begin{aligned}
& \sum_{n=1}^{\infty}(-1)^{n+1}\left[\frac{(2 n+2 a+1)^{p}-(2 n-2 a-1)^{p}}{\left(4 n^{2}-(2 a+1)^{2}\right)^{p}}\right] \\
& \quad=2(-1)^{a} \sum_{n=1}^{\infty} \frac{(-1)^{n+1}}{(2 n-1)^{p}}-\frac{1}{(2 a+1)^{p}}+\sum_{r=1}^{a} \frac{(-1)^{a-r}\left(1+(-1)^{p}\right)}{(2 r-1)^{p}} \\
& \quad=2(-1)^{a} \sum_{n=1}^{\infty} \frac{(-1)^{n+1}}{(2 n-1)^{p}}-\frac{1}{(2 a+1)^{p}}+ \begin{cases}0, & \text { for } p \text { odd } \\
\sum_{r=1}^{a} \frac{2(-1)^{a-r}}{(2 r-1)^{p}}, & \text { for } p \text { even. }\end{cases}
\end{aligned}
$$

For $p=1$ we recover the first result in Table 3.1. For $p=2$

$$
\begin{aligned}
& \sum_{n=1}^{\infty} \frac{(-1)^{n+1} n}{\left(4 n^{2}-(2 a+1)^{2}\right)^{2}} \\
& =\frac{(-1)^{a}}{4(2 a+1)} \sigma(2)-\frac{1}{8(2 a+1)^{3}}+\frac{1}{8(2 a+1)} \sum_{r=1}^{a} \frac{2(-1)^{a-r}}{(2 r-1)^{2}} \\
& =\frac{(-1)^{a}}{4(2 a+1)}\left[1-\Phi\left(-1,2,-\frac{1}{2}\right)\right]-\frac{1}{8(2 a+1)^{3}}+\frac{1}{8(2 a+1)} \sum_{r=1}^{a} \frac{2(-1)^{a-r}}{(2 r-1)^{2}} .
\end{aligned}
$$

For $p=3$,

$$
\sum_{n=1}^{\infty} \frac{(-1)^{n+1}\left(12 n^{2}+(2 a+1)^{2}\right)}{\left(4 n^{2}-(2 a+1)^{2}\right)^{3}}=\frac{(-1)^{a} \pi^{3}}{32(2 a+1)}-\frac{1}{2(2 a+1)^{4}}
$$

and since

$$
A S(a, 3)=\sum_{n=1}^{\infty} \frac{(-1)^{n+1}}{\left(4 n^{2}-(2 a+1)^{2}\right)^{3}}=\frac{(-1)^{a} \pi^{3}}{128(2 a+1)^{3}}+\frac{(-1)^{a} 3 \pi}{32(2 a+1)^{5}}-\frac{1}{2(2 a+1)^{6}},
$$

we can determine

$$
\sum_{n=1}^{\infty} \frac{(-1)^{n+1} n^{2}}{\left(4 n^{2}-(2 a+1)^{2}\right)^{3}}=\frac{(-1)^{a} \pi^{3}}{2^{9} \cdot(2 a+1)}-\frac{(-1)^{a} \pi}{2^{7} \cdot(2 a+1)^{3}} .
$$


In a similar fashion we can add (2.2) to (2.4) to obtain

$$
\begin{aligned}
& \sum_{n=1}^{\infty} \frac{(2 n+2 a+1)^{p}+(2 n-2 a-1)^{p}}{\left(4 n^{2}-(2 a+1)^{2}\right)^{p}} \\
& =2 \delta(p)-\frac{1}{(2 a+1)^{p}}+\sum_{r=1}^{a} \frac{(-1)^{p}-1}{(2 a+1-2 r)^{p}} \\
& =2^{1-p} \Phi\left(1, p,-\frac{1}{2}\right)-\frac{1}{(2 a+1)^{p}}+ \begin{cases}0, & \text { for } p \text { even, } \\
\sum_{r=1}^{a} \frac{-2}{2 a+1-2 r}, & \text { for } p \text { odd. }\end{cases}
\end{aligned}
$$

For $p=3$ we obtain

$$
\sum_{n=1}^{\infty} \frac{4 n^{3}+3 n(2 a+1)^{2}}{\left(4 n^{2}-(2 a+1)^{2}\right)^{3}}=\frac{7}{16} \zeta(3)-\frac{1}{4(2 a+1)^{3}}-\frac{1}{2} \sum_{r=1}^{a} \frac{1}{(2 a+1-2 r)},
$$

and for $a=1$, (4.26) reduces to

$$
\sum_{n=1}^{\infty} \frac{4 n^{3}+27 n}{\left(4 n^{2}-9\right)^{3}}=\frac{7}{16} \zeta(3)-\frac{55}{108}
$$

from which we may obtain

$$
\sum_{n=1}^{\infty}\left(\frac{4 n}{4 n^{2}-9}\right)^{3}=\frac{7}{4} \zeta(3)-\frac{53}{54}
$$

For $p=4$, we have

$$
\sum_{n=1}^{\infty} \frac{16 n^{4}+24 n^{2}(2 a+1)^{2}+(2 a+1)^{4}}{\left(4 n^{2}-(2 a+1)^{2}\right)^{4}}=\frac{\pi^{4}}{96}-\frac{1}{2(2 a+1)^{4}},
$$

and utilising the fourth entry in Table 2.1, results in

$$
\sum_{n=1}^{\infty} \frac{2 n^{4}+3 n^{2}(2 a+1)^{2}}{\left(4 n^{2}-(2 a+1)^{2}\right)^{4}}=\frac{7 \pi^{4}}{3 \cdot 2^{11}}-\frac{5 \pi^{2}}{2^{10} \cdot(2 a+1)^{2}} .
$$

As a final note we can see that

$$
\begin{aligned}
S(a, p)+A S(a, p) & =\sum_{n=1}^{\infty} \frac{1}{\left(4 n^{2}-(2 a+1)^{2}\right)^{p}}+\sum_{n=1}^{\infty} \frac{(-1)^{n+1}}{\left(4 n^{2}-(2 a+1)^{2}\right)^{p}} \\
& =\sum_{n=1}^{\infty} \frac{1}{\left((4 n-2)^{2}-(2 a+1)^{2}\right)^{p}} \\
& =\frac{(-1)^{p}}{2} \sum_{k=1}^{p}\left|A_{p-k+1}\right|\left[\left\{1+(-1)^{k}\right\} \delta(k)-(-1)^{a}\left\{1-(-1)^{k}\right\} \sigma(k)\right],
\end{aligned}
$$


and similarly

$$
\begin{aligned}
S(a, p)-A S(a, p) & =\sum_{n=1}^{\infty} \frac{1}{\left(4 n^{2}-(2 a+1)^{2}\right)^{p}}-\sum_{n=1}^{\infty} \frac{(-1)^{n+1}}{\left(4 n^{2}-(2 a+1)^{2}\right)^{p}} \\
& =\sum_{n=1}^{\infty} \frac{1}{\left((4 n)^{2}-(2 a+1)^{2}\right)^{p}} \\
& =\frac{-(-1)^{p}}{2(2 a+1)^{2 p}}+\frac{(-1)^{p}}{2} \sum_{k=1}^{p}\left|A_{p-k+1}\right| \\
& \times\left[\left\{1+(-1)^{k}\right\} \delta(k)+(-1)^{a}\left\{1-(-1)^{k}\right\} \sigma(k)\right] .
\end{aligned}
$$

For $p=4$ we find that

$$
\begin{aligned}
& \sum_{n=1}^{\infty} \frac{1}{\left((4 n-2)^{2}-(2 a+1)^{2}\right)^{4}} \\
& \quad=\frac{\pi^{4}}{3 \cdot 2^{9}(2 a+1)^{4}}-\frac{(-1)^{a} \pi^{3}}{2^{8} \cdot(2 a+1)^{5}}+\frac{5 \pi^{2}}{2^{8}(2 a+1)^{6}}-\frac{(-1)^{a} 5 \pi}{2^{7} \cdot(2 a+1)^{7}}, \\
& \sum_{n=1}^{\infty} \frac{1}{\left(16 n^{2}-(2 a+1)^{2}\right)^{4}} \\
& \quad=\frac{\pi^{4}}{3 \cdot 2^{9}(2 a+1)^{4}}+\frac{(-1)^{a} \pi^{3}}{2^{8} \cdot(2 a+1)^{5}}+\frac{5 \pi^{2}}{2^{8}(2 a+1)^{6}}+\frac{(-1)^{a} 5 \pi}{2^{7} \cdot(2 a+1)^{7}}-\frac{1}{2(2 a+1)^{8}} .
\end{aligned}
$$

All of the analysis in this paper can be done with the series,

$$
\sum_{n=1}^{\infty} \frac{1}{\left(4 n^{2}-\alpha^{2}\right)^{p}}, \quad \sum_{n=1}^{\infty} \frac{(-1)^{n+1}}{\left(4 n^{2}-\alpha^{2}\right)^{p}},
$$

and excluding the $2 n-\alpha=0$ term, rather than the series (1.1) and (1.4).

ACKNOWLEDGMENT. I would like to thank the anonymous referee for some useful comments on this paper.

\section{REFERENCES}

[1] M. Abramowitz and I. A. Stegun, Handbook of Mathematical Functions, Dover Publications Inc., New York, 1970.

[2] E. P. Adams, Smithsonian Mathematical Formulae, Smithsonian Institute, Washington, 1922.

[3] T. J. I'A. Bromwich, An Introduction to the Theory of Infinite Series, MacMillan and Co. Ltd., London, 1949.

[4] P. Flajolet and B. Salvy, Euler sums and contour integral representations, Experiment. Math. 7 (1998), no. 1, 15-35.

[5] G. Gasper and M. Rahman, Basic Hypergeometric Series, Encyclopedia of Mathematics and its Applications, vol. 35, Cambridge University Press, Cambridge, 1990.

[6] I. S. Gradshteyn and I. M. Ryzhik, Table of Integrals, Series and Products, Academic Press, New York, 1994.

[7] L. B. W. Jolley, Summation of Series, 2nd ed., Dover Books on Advanced Mathematics, Dover Publications Inc., New York, 1961. 
[8] H. Lee and J. Choi, Series associated with the zeta function, Inequality Theory and Applications. Vol. I, Nova Science Publishers, Inc, New York, 2001, pp. 193-208.

[9] J. F. Lin, Elementary solvabilities of $\sum_{n=1}^{\infty} \frac{1}{n^{2 m}}$, Math. Practice Theory 29 (1999), no. 3, 1418.

[10] Q. M. Luo, B.-N. Guo, and F. Qi, On evaluation of Riemann zeta function $\zeta(s)$, Adv. Stud. Contemp. Math. (Kyungshang) 7 (2003), no. 2, 135-144.

Anthony Sofo: School of Computer Science and Mathematics, Victoria University of Technology, P.O. Box 14428, Victoria 8001, Australia.

E-mail address: sofo@csm.vu.edu. au 


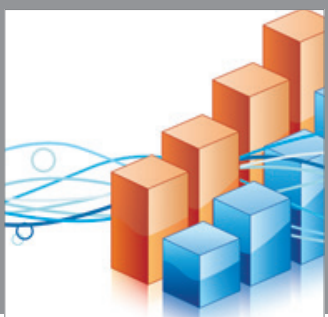

Advances in

Operations Research

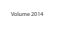

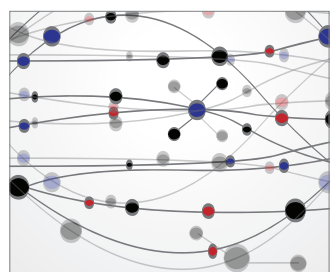

\section{The Scientific} World Journal
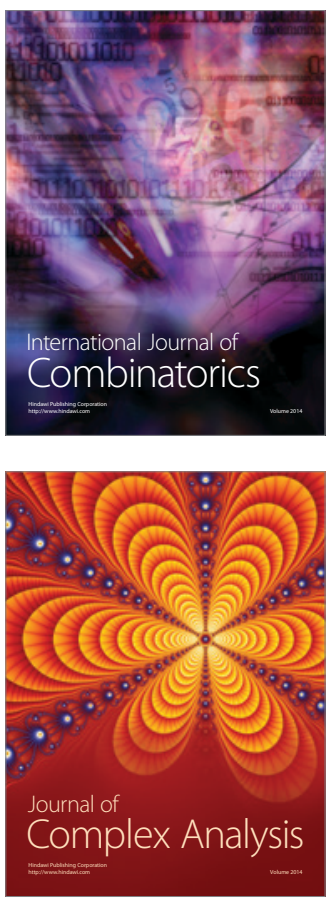

International Journal of

Mathematics and

Mathematical

Sciences
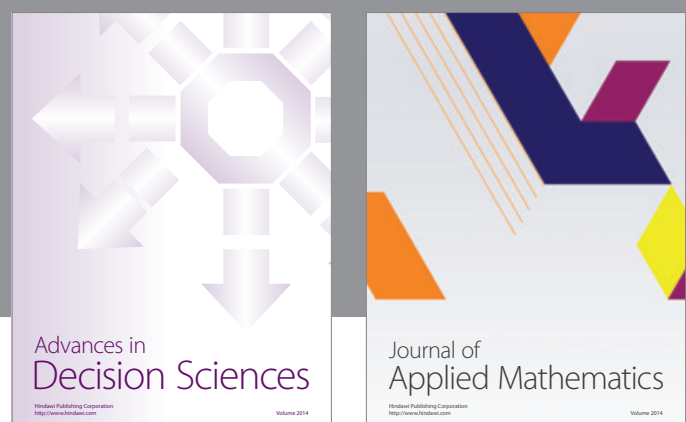

Journal of

Applied Mathematics
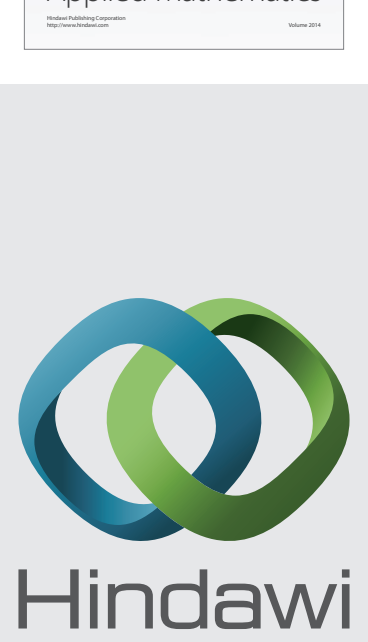

Submit your manuscripts at http://www.hindawi.com
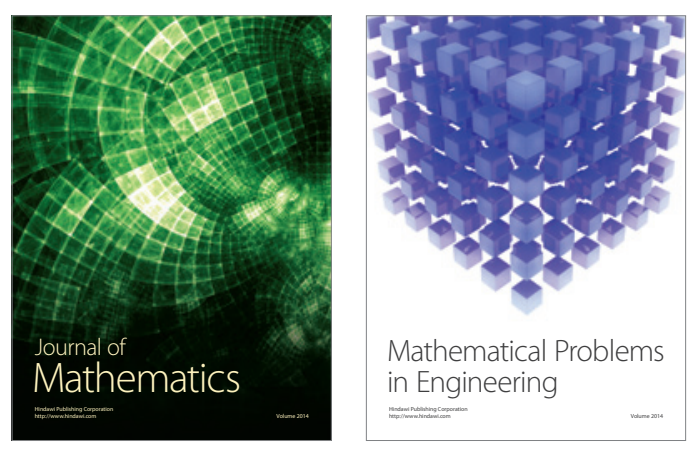

Mathematical Problems in Engineering
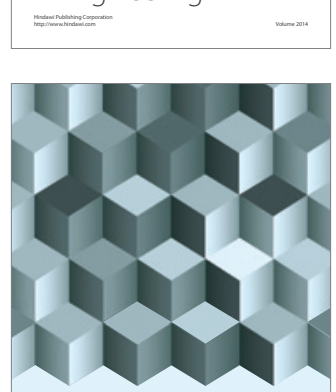

Journal of

Function Spaces
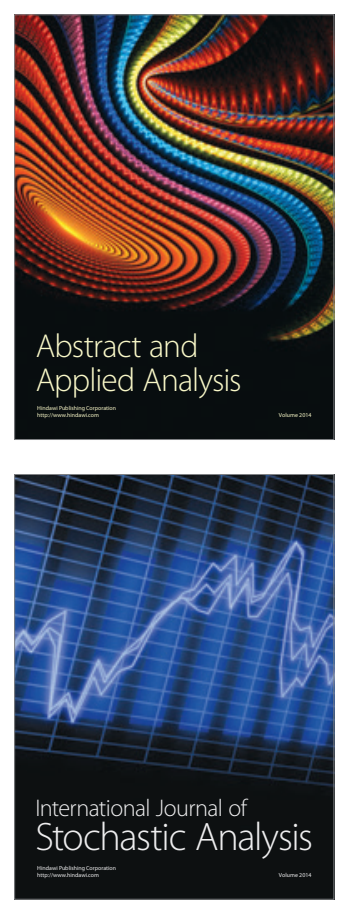

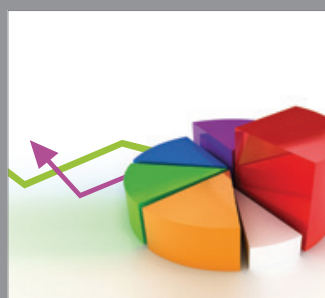

ournal of

Probability and Statistics

Promensencen
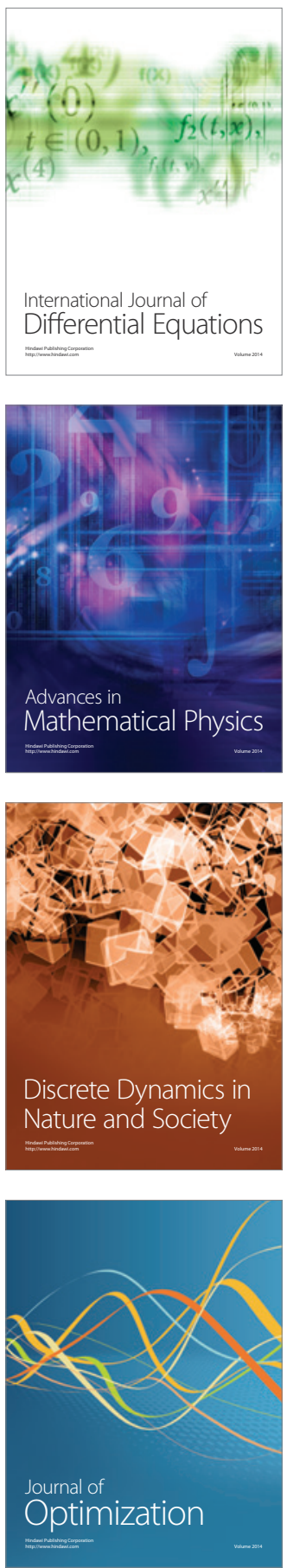